\title{
AN INVERSE GAMMA ACTIVITY TIME PROCESS WITH NONINTEGER PARAMETERS AND A SELF-SIMILAR LIMIT
}

\author{
RICHARD FINLAY $* * *$ AND \\ EUGENE SENETA, ${ }^{* * *}$ University of Sydney \\ DINGCHENG WANG, ${ }^{* * * *}$ Australian National University and Nanjing Audit University
}

\begin{abstract}
We construct a process with inverse gamma increments and an asymptotically self-similar limit. This construction supports the use of long-range-dependent $t$ subordinator models for actual financial data as advocated in Heyde and Leonenko (2005), in that it allows for noninteger-valued model parameters, as is found empirically in model estimation from data.

Keywords: Inverse gamma process; $t$-distribution; subordinator model; long-range dependence; self similarity

2010 Mathematics Subject Classification: Primary 60G10

Secondary 60G18; 62P20
\end{abstract}

\section{Introduction}

Heyde and Leonenko (2005) and Finlay and Seneta (2006) respectively constructed discrete time $t$ and variance-gamma-distributed (VG-distributed) subordinator models which exhibit long-range dependence (LRD) of squared returns, a desirable property for asset price models. In particular, they considered models of the form

$$
\log \left(P_{t}\right)=\log \left(P_{0}\right)+\mu t+\theta T_{t}+\sigma B\left(T_{t}\right),
$$

where $P_{t}$ is some asset price, $B(t)$ is Brownian motion, $\mu, \theta \in \mathbb{R}$, and $\sigma>0$. The LRD of squared returns comes from asymptotically self-similar inverse gamma (for the $t$-model) and gamma (for the VG model) based 'activity time' $\left\{T_{t}\right\}$ processes, and in particular is driven by the LRD of the increment processes, denoted by $\tau(t)=T_{t}-T_{t-1}, t=1,2, \ldots$. Here a continuous-time process $\left\{Y_{t}\right\}$ is said to be self-similar with parameter $H$ if $Y_{c t} \stackrel{\text { D }}{=} c^{H} Y_{t}$; LRD of a discrete-time stationary process with ultimately nonnegative autocorrelations $\left\{\gamma_{k}\right\}$ is said to hold if $\sum_{k=1}^{\infty} \gamma_{k}=\infty$; and the activity time $T_{t}$, as opposed to the standard clock time $t$, is the increasing stochastic process over which security prices are taken to evolve. The $\left\{T_{t}\right\}$ processes are scaled such that their increments over unit time have unit expectation, with the $\tau(t)$ taken to be inverse (or reciprocal) gamma $R \Gamma(v / 2, v / 2-1)$ and gamma $\Gamma(v / 2, v / 2)$ distributed, respectively having probability density functions

$$
f_{R \Gamma}(x)=\frac{(v / 2-1)^{v / 2}}{\Gamma(v / 2)} x^{-v / 2-1} \mathrm{e}^{(1-v / 2) / x}
$$

Received 18 January 2011; revision received 16 November 2011.

* Postal address: School of Mathematics and Statistics F07, University of Sydney, NSW 2006, Australia.

**Email address: richardf@maths.usyd.edu.au

*** Email address: eseneta@maths.usyd.edu.au

**** Email address: wangdc63@126.com 
and

$$
f_{\Gamma}(x)=\frac{(\nu / 2)^{v / 2}}{\Gamma(\nu / 2)} x^{\nu / 2-1} \mathrm{e}^{-(\nu / 2) x}
$$

for $x>0$. Here if $V \sim \Gamma(\alpha, \beta)$ is gamma distributed (so that $B(V)$ has the VG distribution) then $1 / V \sim R \Gamma(\alpha, \beta)$ is inverse gamma distributed (and $B(1 / V)$ has the $t$-distribution).

The activity time processes constructed in Heyde and Leonenko (2005) and Finlay and Seneta (2006) are restricted to integer $v$ however, a condition not consistent with estimation with actual data. Finlay and Seneta (2007) extended $\tau(t)$, the gamma process from which the activity time process $T_{t}=\sum_{k=1}^{t} \tau(k)$ is built, to allow for noninteger $v$. In this note we do likewise for the inverse gamma process.

\section{The inverse gamma increment process $\tau(t)$}

Let $\left\{X_{t}, t \in \mathbb{N}\right\}$ be the stationary gamma process constructed in Finlay and Seneta (2007, Section 2), which, for $\iota=(v-[v]) / 2([v]$ denoting the integer part of $v)$, has a $\Gamma\left(\iota, \frac{1}{2}\right)$ distribution and bivariate characteristic function given by

$$
\mathrm{E}\left(\mathrm{e}^{u \mathrm{i} X_{t}+v \mathrm{i} X_{t+s}}\right)=((1-2 \mathrm{i} u)(1-2 \mathrm{i} v))^{-\iota+\iota \rho_{2}(s)}(1-2 \mathrm{i}(u+v))^{-\iota \rho_{2}(s)} .
$$

Here $\rho_{2}(s)$ is the autocorrelation function of $\left\{X_{t}\right\}$, and can be taken as any function that satisfies Assumption 1 below.

Assumption 1. $\rho_{2}(s)$ is a function on $\{0,1,2, \ldots\}$ satisfying $\rho_{2}(0)=1, \rho_{2}(s) \geq 0, \rho_{2}(s)-$ $\rho_{2}(s+1) \geq 0$, and $\rho_{2}(s)-2 \rho_{2}(s+1)+\rho_{2}(s+2) \geq 0$.

The construction in Finlay and Seneta (2007, Section 2) has the consequence of providing an indirect proof that any function $\rho_{2}(s)$ satisfying Assumption 1 is an autocorrelation function of a strictly stationary process. The same issue is discussed more generally in Finlay et al. (2011).

Next, for $\mathbb{N}=\{1,2,3, \ldots\}$, let $\left\{\eta_{i}(t), t \in \mathbb{N}\right\}, i=1, \ldots,[v]$, with noninteger $v>4$, be independent and identically distributed stationary Gaussian processes with zero mean, unit variance, and autocorrection function $\rho_{1}(s), s \in \mathbb{N}$. We assume that $\rho_{1}(s)$ is of the form

$$
\rho_{1}(s)=\left(1+\omega|s|^{\alpha}\right)^{(H-1) / \alpha}
$$

for $\omega>0,0<\alpha \leq 2$, and $\frac{1}{2}<H<1$. That is, we assume that $\rho_{1}(s)$ belongs to the Cauchy family of autocorrection functions discussed in Gneiting (2000).

Define the independent stationary processes $\left\{\chi_{t}^{2}\right\}$ and $\left\{V_{t}\right\}, t \in \mathbb{N}$, by

$$
\chi_{t}^{2}=\frac{\eta_{1}^{2}(t)+\cdots+\eta_{[v]}^{2}(t)}{2} \sim \Gamma\left(\frac{[v]}{2}, 1\right), \quad V_{t}=\frac{X_{t}}{2} \sim \Gamma(\iota, 1) .
$$

Then $\left(\chi_{t}^{2}+V_{t}\right) \sim \Gamma(v / 2,1)$ and $\operatorname{cor}\left(\chi_{t}^{2}+V_{t}, \chi_{t+s}^{2}+V_{t+s}\right)=([v] / v) \rho_{1}^{2}(s)+(1-[v] / v)$ $\rho_{2}(s)$.

We proceed to construct our inverse gamma activity time process $\left\{T_{t}\right\}$ as

$$
T_{t}=\sum_{k=1}^{t} \tau(k), \quad \tau(t)=\frac{v / 2-1}{\chi_{t}^{2}+V_{t}} \sim R \Gamma\left(\frac{v}{2}, \frac{v}{2}-1\right) .
$$

This construction ensures that the increments of the activity time process $\left\{T_{t}\right\}$ are inverse gamma distributed and that $\mathrm{E}(\tau(t))=1$. 
Now, from Leonenko (1999, Chapter 2) for example, for $p(x)=x^{[v] / 2-1} \mathrm{e}^{-x} / \Gamma([\nu] / 2)$, the density function of $\chi_{t}^{2}$, we can define a complete orthogonal system of functions $\left\{e_{k}(x)\right\}_{k=0}^{\infty}$ in the Hilbert space $\mathcal{L}_{2}((0, \infty), p(x) \mathrm{d} x)$ by $e_{0}(x) \equiv 1$ and, for $k>0$,

$$
e_{k}(x)=L_{k}^{[\nu] / 2-1}(x)\left(\frac{k ! \Gamma([\nu] / 2)}{\Gamma([\nu] / 2+k)}\right)^{1 / 2}, \quad \text { where } \quad L_{k}^{\beta}(x)=\frac{1}{k !} x^{-\beta} \mathrm{e}^{x} \frac{\mathrm{d}^{k}}{\mathrm{~d} x^{k}}\left\{x^{\beta+k} \mathrm{e}^{-x}\right\}
$$

are the generalised Laguerre polynomials of index $\beta$. That is, for $G(x) \in \mathcal{L}_{2}((0, \infty), p(x) \mathrm{d} x)$,

$$
G\left(\chi_{t}^{2}\right)=\sum_{k=0}^{\infty} C_{k} e_{k}\left(\chi_{t}^{2}\right), \quad \text { where } \quad C_{k}=\int_{0}^{\infty} G(x) p(x) e_{k}(x) \mathrm{d} x,
$$

and $\sum_{k=0}^{\infty} C_{k}^{2}=\int_{0}^{\infty} G(x)^{2} p(x) \mathrm{d} x<\infty$. For $\delta_{k}^{m}$, the Kronecker symbol, we also have the useful property

$$
\mathrm{E}\left(e_{k}\left(\chi_{t}^{2}\right) e_{m}\left(\chi_{t+s}^{2}\right)\right)=\delta_{k}^{m} \rho_{1}^{2 k}(s),
$$

so, for example, $\operatorname{cov}\left(G\left(\chi_{t}^{2}\right), G\left(\chi_{t+s}^{2}\right)\right)=\sum_{k=1}^{\infty} C_{k}^{2} \rho_{1}^{2 k}(s)$.

Now, for fixed $y>0$ and $v>4,(v / 2-1) /(x+y) \in \mathcal{L}_{2}((0, \infty), p(x) \mathrm{d} x)$, so, given $V_{t}$, we can expand $(v / 2-1) /\left(\chi_{t}^{2}+V_{t}\right)$ as $\sum_{k=0}^{\infty} C_{k}\left(V_{t}\right) e_{k}\left(\chi_{t}^{2}\right)$. From (5),

$$
\infty>\mathrm{E}\left(\frac{v / 2-1}{\chi_{t}^{2}+V_{t}}\right)^{2}=\mathrm{E}\left(\mathrm{E}\left(\left(\sum_{k=0}^{\infty} C_{k}\left(V_{t}\right) e_{k}\left(\chi_{t}^{2}\right)\right)^{2} \mid V_{t}\right)\right)=\sum_{k=0}^{\infty} \mathrm{E}\left(C_{k}^{2}\left(V_{t}\right)\right) .
$$

Given the above,

$$
\begin{aligned}
\mathrm{E}\left(\frac{v / 2-1}{\chi_{t}^{2}+V_{t}}-\sum_{k=0}^{n} C_{k}\left(V_{t}\right) e_{k}\left(\chi_{t}^{2}\right)\right)^{2} & =\mathrm{E}\left(\mathrm{E}\left(\left(\sum_{k=n+1}^{\infty} C_{k}\left(V_{t}\right) e_{k}\left(\chi_{t}^{2}\right)\right)^{2} \mid V_{t}\right)\right) \\
& =\sum_{k=n+1}^{\infty} \mathrm{E}\left(C_{k}^{2}\left(V_{t}\right)\right),
\end{aligned}
$$

which goes to 0 as $n \rightarrow \infty$. Thus, we have (in terms of mean-square convergence)

$$
\tau(t)=\frac{v / 2-1}{\chi_{t}^{2}+V_{t}}=\sum_{k=0}^{\infty} C_{k}\left(V_{t}\right) e_{k}\left(\chi_{t}^{2}\right) \sim R \Gamma\left(\frac{v}{2}, \frac{v}{2}-1\right) .
$$

Our inverse gamma activity time process $\left\{T_{t}\right\}$ will be constructed as the sum of these $\tau(t)$, as given in (2).

\section{Main result}

Consider the normalized inverse gamma activity time process given by

$$
\frac{1}{n^{H}}\left(T_{[n t]}-[n t]\right)=\frac{1}{n^{H}} \sum_{k=1}^{[n t]}(\tau(k)-1)
$$

where in what follows we will hold $v$ fixed and let $n \rightarrow \infty$, and, therefore, normalise by $n^{H}$ (note that $v$ appears in the limit process - see (15) below-but not in a form that 
requires normalisation). From (6) and for $\bar{C}_{1}=\mathrm{E}\left(C_{1}\left(V_{t}\right)\right)$, we can decompose this process into the four terms

$$
\begin{aligned}
T_{[n t]}-[n t]= & \underbrace{\sum_{m=1}^{[n t]} \sum_{k=2}^{\infty}\left(C_{k}\left(V_{m}\right) e_{k}\left(\chi_{m}^{2}\right)\right)}_{A_{n t}}+\underbrace{\sum_{m=1}^{[n t]}\left(\left(C_{1}\left(V_{m}\right)-\bar{C}_{1}\right) e_{1}\left(\chi_{m}^{2}\right)\right)}_{B_{n t}} \\
& +\underbrace{\sum_{m=1}^{[n t]}\left(\bar{C}_{1} e_{1}\left(\chi_{m}^{2}\right)\right)}_{C_{n t}}+\underbrace{\sum_{m=1}^{[n t]}\left(C_{0}\left(V_{m}\right)-1\right)}_{D_{n t}},
\end{aligned}
$$

where the equality holds in the sense of mean-square convergence. We then have the following result.

Theorem 1. For any $\rho_{2}(s)$ satisfying Assumption 1 and further satisfying $\sum_{s=1}^{\infty} \rho_{2}(s)<\infty$, $A_{n t} / n^{H}, B_{n t} / n^{H}$, and $D_{n t} / n^{H}$ converge in mean square to 0 as $n \rightarrow \infty$ and $C_{n t} / n^{H}$ converges weakly to the sum of $[v]$ independent copies of the H self-similar Rosenblatt process.

Theorem 1 is the main result of this paper and implies that the process given in (7) converges weakly to a sum of self-similar Rosenblatt processes (the Rosenblatt process is discussed in greater detail below). It complements the result of Finlay and Seneta (2007) for gamma activity time processes and supports the use of long-range-dependent $t$ subordinator models for actual financial data as advocated in Heyde and Leonenko (2005), in that it proves that activity time processes $\left\{T_{t}\right\}$ which display asymptotic self-similarity and have inverse gamma distributed increments (and so imply asset price increments with the $t$-distribution) exist.

Proof of Theorem 1. First consider

$$
A_{n t}=\sum_{m=1}^{[n t]} \sum_{k=2}^{\infty}\left(C_{k}\left(V_{m}\right) e_{k}\left(\chi_{m}^{2}\right)\right) .
$$

For $k>0, \mathrm{E}\left(e_{k}\left(\chi_{m}^{2}\right)\right)=0$, so $\mathrm{E}\left(A_{n t}\right)=0$. Then, since the $\left\{V_{t}\right\}$ and $\left\{\chi_{t}^{2}\right\}$ are independent, using (5), the Cauchy-Schwarz inequality, and the functional form of $\rho_{1}^{2}(s)$, we have, irrespective of $\rho_{2}(s)$,

$$
\begin{aligned}
\operatorname{var}\left(\frac{A_{n t}}{n^{H}}\right) & =\frac{1}{n^{2 H}} \sum_{k=2}^{\infty} \sum_{m=1}^{[n t]} \sum_{s=1}^{[n t]} \mathrm{E}\left(C_{k}\left(V_{m}\right) C_{k}\left(V_{s}\right)\right) \rho_{1}^{2 k}(|s-m|) \\
& \leq \sum_{k=2}^{\infty} \mathrm{E}\left(C_{k}^{2}\left(V_{0}\right)\right) \frac{1}{n^{2 H}} \sum_{m=1}^{[n t]} \sum_{s=1}^{[n t]} \rho_{1}^{4}(|s-m|) \\
& =k_{1}^{*}\left(\frac{[n t]}{n^{2 H}}+\frac{2}{n^{2 H}} \sum_{s=1}^{[n t]-1}([n t]-s)\left(1+\omega|s|^{\alpha}\right)^{4(H-1) / \alpha}\right)
\end{aligned}
$$

for $k_{1}^{*}=\sum_{k=2}^{\infty} \mathrm{E}\left(C_{k}^{2}\left(V_{0}\right)\right)<\infty$. Here the last two lines follow since $\rho_{1}^{2 k}(|s-m|) \leq \rho_{1}^{4}(\mid s-$ $m \mid$ ) for $k \geq 2$, and, for any positive integer $a$ and any function $I$,

$$
\sum_{m=1}^{a} \sum_{s=1}^{a} I(|s-m|)=a I(0)+2 \sum_{s=1}^{a-1}(a-s) I(s) .
$$


Now $[n t] / n^{2 H} \rightarrow 0$ for $\frac{1}{2}<H<1$, and, for $\frac{1}{2}<H<1, H \neq \frac{3}{4}$, and $k_{2}^{*}<\infty$ a constant, we have

$$
\begin{aligned}
\frac{2}{n^{2 H}} \sum_{s=1}^{[n t]-1}([n t]-s)\left(1+\omega|s|^{\alpha}\right)^{4(H-1) / \alpha} & <\omega^{4(H-1) / \alpha} \frac{[n t]}{n^{2 H}} \sum_{s=1}^{[n t]} s^{4(H-1)} \\
& \leq k_{2}^{*}(n t)^{1-2 H} \int_{1}^{n t} s^{4(H-1)} \mathrm{d} s \\
& =k_{2}^{*}\left(\frac{(n t)^{2 H-2}-(n t)^{1-2 H}}{4 H-3}\right),
\end{aligned}
$$

which is positive but goes to 0 as $n \rightarrow \infty$. Here (9) follows since, for $s \geq 1, \alpha, \gamma, \omega>0$, and $\frac{1}{2}<H<1$,

$$
\left(1+\omega s^{\alpha}\right)^{\gamma(H-1) / \alpha}<\omega^{\gamma(H-1) / \alpha} s^{\gamma(H-1)} .
$$

For $H=\frac{3}{4}$, (10) becomes

$$
k_{2}^{*}(n t)^{-1 / 2} \int_{1}^{n t} \frac{\mathrm{d} s}{s}=k_{2}^{*}(n t)^{-1 / 2} \log (n t) \rightarrow 0 \quad \text { as } n \rightarrow \infty .
$$

Hence, $\operatorname{var}\left(A_{n t} / n^{H}\right) \rightarrow 0$ as $n \rightarrow \infty$ for $\frac{1}{2}<H<1$ and so $A_{n t} / n^{H}$ converges to 0 in mean square for each $t$, irrespective of $\rho_{2}(s)$.

Next consider

$$
B_{n t}=\sum_{m=1}^{[n t]}\left(\left(C_{1}\left(V_{m}\right)-\bar{C}_{1}\right) e_{1}\left(\chi_{m}^{2}\right)\right) .
$$

First note that, for $G(x)=(v / 2-1) /(x+y)$ and $p(x)=x^{[v] / 2-1} \mathrm{e}^{-x} / \Gamma([v] / 2)$, we can compute $C_{1}=C_{1}(y)=\int_{0}^{\infty} G(x) p(x) e_{1}(x) \mathrm{d} x$ from (4) as

$$
C_{1}(y)=\left(\frac{v}{2}-1\right) \sqrt{\frac{[\nu]}{2}} \int_{1}^{\infty} \mathrm{e}^{y(1-t)}\left(t^{-[v] / 2}-t^{-[v] / 2-1}\right) \mathrm{d} t .
$$

Then, from (1), since $V_{m}(1-t)=X_{m}((1-t) / 2)$, we have, for $t, u \geq 1$,

$$
0 \leq \mathrm{E}\left(\mathrm{e}^{V_{m}(1-t)+V_{s}(1-u)}\right)=(t u)^{-\iota+\iota \rho_{2}(|s-m|)}(t+u-1)^{-\iota \rho_{2}(|s-m|)} \leq(t u)^{-\imath+\iota \rho_{2}(|s-m|)} .
$$

Hence,

$$
\begin{aligned}
& 0 \leq \mathrm{E}\left(C_{1}\left(V_{m}\right) C_{1}\left(V_{s}\right)\right) \\
& =\left(\frac{v}{2}-1\right)^{2} \frac{[v]}{2} \int_{1}^{\infty} \int_{1}^{\infty} \mathrm{E}\left(\mathrm{e}^{V_{m}(1-t)+V_{s}(1-u)}\right)\left(\frac{1}{t^{[v] / 2}}-\frac{1}{t^{[v] / 2+1}}\right) \\
& \times\left(\frac{1}{u^{[v] / 2}}-\frac{1}{u^{[v] / 2+1}}\right) \mathrm{d} t \mathrm{~d} u \\
& \leq\left(\frac{v}{2}-1\right)^{2} \frac{[\nu]}{2} \int_{1}^{\infty} \int_{1}^{\infty}(t u)^{-\iota+\iota \rho_{2}(|s-m|)}\left(\frac{1}{t^{[v] / 2}}-\frac{1}{t^{[v] / 2+1}}\right)\left(\frac{1}{u^{[v] / 2}}-\frac{1}{u^{[v] / 2+1}}\right) \mathrm{d} t \mathrm{~d} u \\
& =\left(\frac{v}{2}-1\right)^{2} \frac{[\nu]}{2}\left(\frac{1}{\left([v] / 2+\iota-\iota \rho_{2}(|s-m|)-1\right)^{2}}+\frac{1}{\left([v] / 2+\iota-\iota \rho_{2}(|s-m|)\right)^{2}}\right. \\
& \left.-\frac{2}{\left([v] / 2+\iota-\iota \rho_{2}(|s-m|)-1\right)\left([v] / 2+\iota-\iota \rho_{2}(|s-m|)\right)}\right) .
\end{aligned}
$$


Similarly,

$$
\begin{aligned}
\left(\bar{C}_{1}\right)^{2} & =\left(\mathrm{E}\left(C_{1}\left(V_{m}\right)\right)\right)^{2} \\
& =\left(\frac{v}{2}-1\right)^{2} \frac{[\nu]}{2}\left(\int_{1}^{\infty} \mathrm{E}\left(\mathrm{e}^{V_{m}(1-t)}\right)\left(\frac{1}{t^{[\nu] / 2}}-\frac{1}{t^{[v] / 2+1}}\right) \mathrm{d} t\right)^{2} \\
& =\left(\frac{v}{2}-1\right)^{2} \frac{[\nu]}{2}\left(\int_{1}^{\infty} t^{-\iota}\left(\frac{1}{t^{[\nu] / 2}}-\frac{1}{t^{[v] / 2+1}}\right) \mathrm{d} t\right)^{2} \\
& =\left(\frac{v}{2}-1\right)^{2} \frac{[\nu]}{2}\left(\frac{1}{([v] / 2+\iota-1)^{2}}+\frac{1}{([v] / 2+\iota)^{2}}-\frac{2}{([v] / 2+\iota-1)([v] / 2+\iota)}\right) .
\end{aligned}
$$

Now, from (12), (13), and (14),

$$
\begin{gathered}
\mathrm{E}\left(C_{1}\left(V_{m}\right) C_{1}\left(V_{s}\right)\right)-\left(\bar{C}_{1}\right)^{2} \\
=\left(\frac{v}{2}-1\right)^{2} \frac{[\nu]}{2} \int_{1}^{\infty} \int_{1}^{\infty}(t u)^{-\iota}\left(\left(\frac{t u}{t+u-1}\right)^{\iota \rho_{2}(|s-m|)}-1\right)\left(\frac{1}{t^{[\nu] / 2}}-\frac{1}{t^{[\nu] / 2+1}}\right) \\
\times\left(\frac{1}{u^{[\nu] / 2}}-\frac{1}{u^{[\nu] / 2+1}}\right) \mathrm{d} t \mathrm{~d} u .
\end{gathered}
$$

Thus, $\mathrm{E}\left(C_{1}\left(V_{m}\right) C_{1}\left(V_{s}\right)\right)-\left(\bar{C}_{1}\right)^{2} \geq 0$ since $t, u \geq 1$ and so $t u \geq t+u-1$. Then, for $a>b>0$ and $b<1$,

$$
0 \leq \frac{1}{(a-b)^{2}}-\frac{1}{a^{2}}=b\left(\frac{2 a-b}{a^{2}(a-b)^{2}}\right) \leq b\left(\frac{2 a}{a^{2}(a-1)^{2}}\right),
$$

so

$$
\begin{aligned}
0 & \leq \mathrm{E}\left(\left(C_{1}\left(V_{m}\right)-\bar{C}_{1}\right)\left(C_{1}\left(V_{s}\right)-\bar{C}_{1}\right)\right) \\
& =\mathrm{E}\left(C_{1}\left(V_{m}\right) C_{1}\left(V_{s}\right)\right)-\left(\bar{C}_{1}\right)^{2} \\
& \leq\left(\frac{v}{2}-1\right)^{2} \frac{[\nu]}{2}\left(\frac{1}{\left([\nu] / 2+\iota-\iota \rho_{2}(|s-m|)-1\right)^{2}}-\frac{1}{([\nu] / 2+\iota-1)^{2}}\right. \\
& \left.\quad+\frac{1}{\left([v] / 2+\iota-\iota \rho_{2}(|s-m|)\right)^{2}}-\frac{1}{([v] / 2+\iota)^{2}}\right) \\
& \leq k_{3}^{*} \rho_{2}(|s-m|)
\end{aligned}
$$

for $k_{3}^{*}$ a constant. Hence, for $B_{n t}=\sum_{m=1}^{[n t]}\left(\left(C_{1}\left(V_{m}\right)-\bar{C}_{1}\right) e_{1}\left(\chi_{m}^{2}\right)\right)$ and any $\rho_{2}(s)$ satisfying Assumption 1 and further satisfying $\sum_{s=1}^{\infty} \rho_{2}(s)<\infty, \mathrm{E}\left(B_{n t}\right)=0$, and using (8),

$$
\begin{aligned}
\operatorname{var}\left(\frac{B_{n t}}{n^{H}}\right) & =\frac{1}{n^{2 H}} \sum_{m=1}^{[n t]} \sum_{s=1}^{[n t]}\left(\mathrm{E}\left(\left(C_{1}\left(V_{m}\right)-\bar{C}_{1}\right)\left(C_{1}\left(V_{s}\right)-\bar{C}_{1}\right)\right) \rho_{1}^{2}(|s-m|)\right) \\
& \leq \frac{k_{3}^{*}}{n^{2 H}} \sum_{m=1}^{[n t]} \sum_{s=1}^{[n t]} \rho_{2}(|s-m|) \\
& =\frac{k_{3}^{*}}{n^{2 H}}\left([n t]+2 \sum_{s=1}^{[n t]-1}([n t]-s) \rho_{2}(s)\right)
\end{aligned}
$$




$$
\begin{aligned}
& <\frac{k_{3}^{*}[n t]}{n^{2 H}}\left(1+2 \sum_{s=1}^{[n t]-1} \rho_{2}(s)\right) \\
& \rightarrow 0 \text { as } n \rightarrow \infty,
\end{aligned}
$$

where the last line follows since $\sum_{s=1}^{\infty} \rho_{2}(s)<\infty$ by assumption.

For $\rho_{2}(s)=\rho_{1}^{2}(s)$, and, therefore, satisfying Assumption 1 but not satisfying $\sum_{s=1}^{\infty} \rho_{2}(s)<$ $\infty$,

$$
\begin{aligned}
\operatorname{var}\left(\frac{B_{n t}}{n^{H}}\right) & =\frac{1}{n^{2 H}} \sum_{m=1}^{[n t]} \sum_{s=1}^{[n t]}\left(\mathrm{E}\left(\left(C_{1}\left(V_{m}\right)-\bar{C}_{1}\right)\left(C_{1}\left(V_{s}\right)-\bar{C}_{1}\right)\right) \rho_{1}^{2}(|s-m|)\right) \\
& \leq \frac{k_{3}^{*}}{n^{2 H}} \sum_{m=1}^{[n t]} \sum_{s=1}^{[n t]} \rho_{1}^{4}(|s-m|) \\
& \rightarrow 0 \quad \text { from earlier. }
\end{aligned}
$$

Thus, in either case $B_{n t} / n^{H}$ converges in mean square to 0 .

Next consider $C_{n t}=\sum_{m=1}^{[n t]}\left(\bar{C}_{1} e_{1}\left(\chi_{m}^{2}\right)\right)$. Evaluating $e_{1}(x)$ from $(3)$ as $\sqrt{2 /[v]}([v] / 2-x)$, we have, from Taqqu (1975, Theorem 6.1 and Proposition 6.1), irrespective of $\rho_{2}(s)$,

$$
\begin{aligned}
\frac{1}{n^{H}} \sum_{m=1}^{[n t]}\left(\bar{C}_{1} e_{1}\left(\chi_{m}^{2}\right)\right) & =\frac{\bar{C}_{1}}{n^{H}} \sum_{m=1}^{[n t]} \sqrt{\frac{2}{[v]}}\left(\frac{[v]}{2}-\chi_{m}^{2}\right) \\
& =\frac{-\bar{C}_{1}}{n^{H} \sqrt{2[v]}} \sum_{j=1}^{[v]} \sum_{m=1}^{[n t]}\left(\eta_{j}^{2}(m)-1\right) \\
& \Rightarrow \frac{-\bar{C}_{1}}{\sqrt{2[v]}} \sum_{j=1}^{[v]} R_{j}(t),
\end{aligned}
$$

where convergence is in the weak sense and each $R_{j}(t)$ is an independent copy of the selfsimilar Rosenblatt process. The Rosenblatt process $R(t)$ is defined in Taqqu (1975); it has strictly stationary increments and is $H$ self-similar, $\mathrm{E}(R(t))=0$, and $\mathrm{E}\left(|R(t)|^{\gamma}\right)<\infty$ for $\gamma \leq 1 / H$; it is separable and almost surely continuous, and the characteristic function of $R(1)$ admits the representation

$$
\phi(u)=\exp \left\{\frac{1}{2} \sum_{k=2}^{\infty} \frac{(2 \mathrm{i} u)^{k}}{(k+1) !} S_{k}\right\}
$$

for

$$
S_{k}=\int_{0}^{1} \cdots \int_{0}^{1} \frac{\mathrm{d} x_{1} \cdots \mathrm{d} x_{k}}{\left(\left|x_{1}-x_{2}\right|\left|x_{2}-x_{3}\right| \cdots\left|x_{k-1}-x_{k}\right|\left|x_{k}-x_{1}\right|\right)^{1-H}},
$$

which is valid for small values of $|u|$.

Finally, consider

$$
D_{n t}=\sum_{m=1}^{[n t]}\left(C_{0}\left(V_{m}\right)-1\right)
$$

For $D_{n t}, C_{0}(y)$ can be evaluated to give

$$
C_{0}(y)=\left(\frac{v}{2}-1\right) \int_{1}^{\infty} \mathrm{e}^{y(1-t)} t^{-[v] / 2} \mathrm{~d} t
$$


Direct calculation shows that $\mathrm{E}\left(C_{0}\left(V_{m}\right)\right)=1$, while $\operatorname{var}\left(C_{0}\left(V_{m}\right)\right)<\infty$ since

$$
\begin{aligned}
\mathrm{E}\left(C_{0}\left(V_{m}\right)^{2}\right) & =\left(\frac{[\nu]}{2}-1\right)^{2} \int_{0}^{\infty} p^{\iota}(y)\left(\int_{1}^{\infty} \mathrm{e}^{y(1-t)} t^{-[v] / 2} \mathrm{~d} t\right)\left(\int_{1}^{\infty} \mathrm{e}^{y(1-s)} s^{-[v] / 2} \mathrm{~d} s\right) \mathrm{d} y \\
& =\left(\frac{[v]}{2}-1\right)^{2} \int_{1}^{\infty} \int_{1}^{\infty}(t s)^{-[v] / 2}\left(\int_{0}^{\infty} p^{\iota}(y) \mathrm{e}^{y(2-t-s)} \mathrm{d} y\right) \mathrm{d} t \mathrm{~d} s \\
& =\left(\frac{[v]}{2}-1\right)^{2} \int_{1}^{\infty} \int_{1}^{\infty}(t s)^{-[v] / 2}(t+s-1)^{([v]-v) / 2} \mathrm{~d} t \mathrm{~d} s \\
& \leq\left(\frac{[v]}{2}-1\right)^{2} \int_{1}^{\infty} \int_{1}^{\infty}(t+s-1)^{-v / 2} \mathrm{~d} t \mathrm{~d} s \\
& =\frac{([v] / 2-1)^{2}}{(v / 2-1)(v / 2-2)} \\
& <\infty
\end{aligned}
$$

where $p^{\iota}(y)=y^{\iota-1} \mathrm{e}^{-y} / \Gamma(\iota)$ is the density function of $V_{m}$ and $\iota=(v-[v]) / 2$ from earlier. Hence, $C_{0}(y) \in \mathcal{L}_{2}\left((0, \infty), p^{\iota}(y) \mathrm{d} y\right)$ and similar to before we can write

$$
C_{0}\left(V_{m}\right)=\sum_{k=0}^{\infty} C_{k}^{\iota} e_{k}^{\iota}\left(V_{m}\right) \text { for } C_{k}^{\iota}=\int_{0}^{\infty} C_{0}(y) p^{\iota}(y) e_{k}^{\iota}(y) \mathrm{d} y,
$$

where

$$
e_{k}^{\iota}(y)=L_{k}^{\iota-1}(y)\left(\frac{k ! \Gamma(\iota)}{\Gamma(\iota+k)}\right)^{1 / 2} \text { and } \sum_{k=0}^{\infty}\left(C_{k}^{\iota}\right)^{2}=\int_{0}^{\infty} C_{0}^{2}(y) p^{\iota}(y) \mathrm{d} y<\infty .
$$

Since $C_{0}^{\iota}=e_{0}^{\iota}(y)=1$,

$$
\frac{1}{n^{H}} D_{n t}=\frac{1}{n^{H}} \sum_{m=1}^{[n t]}\left(C_{0}\left(V_{m}\right)-1\right)=\frac{1}{n^{H}} \sum_{m=1}^{[n t]} \sum_{k=1}^{\infty} C_{k}^{\iota} e_{k}^{\iota}\left(V_{m}\right) .
$$

Now, for $W_{i} \sim \Gamma\left(a_{i}, 1\right), i=1,2,3$, and each $W_{i}$ independent, we have (see Griffiths (1969))

$$
\mathrm{E}\left(e_{k}^{\iota}\left(W_{1}+W_{2}\right) e_{m}^{\iota}\left(W_{2}+W_{3}\right)\right)=\delta_{k}^{m}\left(\frac{\Gamma\left(a_{1}+a_{2}\right) \Gamma\left(a_{2}+a_{3}\right)}{\Gamma\left(a_{1}+a_{2}+k\right) \Gamma\left(a_{2}+a_{3}+k\right)}\right)^{1 / 2} \frac{\Gamma\left(a_{2}+k\right)}{\Gamma\left(a_{2}\right)} .
$$

From (1) however, $\left\{V_{t}, V_{t+s}\right\} \stackrel{\mathrm{D}}{=}\left\{W_{1}+W_{2}, W_{2}+W_{3}\right\}$ for $W_{i}$ as defined above with $a_{1}=a_{3}=$ $\iota-\iota \rho_{2}(s)$ and $a_{2}=\iota \rho_{2}(s)$, so

$$
\mathrm{E}\left(e_{k}^{\iota}\left(V_{t}\right) e_{m}^{\iota}\left(V_{t+s}\right)\right)=\delta_{k}^{m} \rho_{2}(s)\left(\frac{1+\iota \rho_{2}(s)}{1+\iota}\right) \cdots\left(\frac{k-1+\iota \rho_{2}(s)}{k-1+\iota}\right) \leq \delta_{k}^{m} \rho_{2}(s) .
$$

Hence, for any $\rho_{2}(s)$ satisfying Assumption 1 and additionally satisfying $\sum_{s=1}^{\infty} \rho_{2}(s)<\infty$, we have

$$
\operatorname{var}\left(\frac{D_{n t}}{n^{H}}\right) \leq \frac{1}{n^{2 H}}\left(\sum_{k=1}^{\infty}\left(C_{k}^{\iota}\right)^{2}\right) \sum_{m=1}^{[n t]} \sum_{s=1}^{[n t]} \rho_{2}(|m-s|) \rightarrow 0 \quad \text { from earlier. }
$$


For $\rho_{2}(s)=\rho_{1}^{2}(s)$, and, therefore, not satisfying $\sum_{s=1}^{\infty} \rho_{2}(s)<\infty$, we write $D_{n t} / n^{H}$ as

$$
\frac{1}{n^{H}} D_{n t}=\frac{1}{n^{H}} \sum_{m=1}^{[n t]} C_{1}^{\iota} e_{1}^{\iota}\left(V_{m}\right)+\frac{1}{n^{H}} \sum_{m=1}^{[n t]} \sum_{k=2}^{\infty} C_{k}^{\iota} e_{k}^{\iota}\left(V_{m}\right)
$$

Then direct calculation yields $e_{1}^{\iota}(y)=\sqrt{1 / \iota}(\iota-y)$, so

$$
\frac{1}{n^{H}} \sum_{m=1}^{[n t]} C_{1}^{\iota} e_{1}^{\iota}\left(V_{m}\right)=\frac{-C_{1}^{\iota}}{2 \sqrt{\imath n^{H}}} \sum_{m=1}^{[n t]}\left(2 V_{m}-2 \iota\right),
$$

which, from Finlay and Seneta (2007, Theorem 2), converges in probability to 0 . Next, using (8), (11), and (16),

$$
\begin{aligned}
\operatorname{var}\left(\frac{1}{n^{H}} \sum_{m=1}^{[n t]} \sum_{k=2}^{\infty} C_{k}^{\iota} e_{k}^{\iota}\left(V_{m}\right)\right) & \leq \frac{1}{n^{2 H}}\left(\sum_{k=2}^{\infty}\left(C_{k}^{\iota}\right)^{2}\right) \sum_{m=1}^{[n t]} \sum_{s=1}^{[n t]} \rho_{1}^{2}(|m-s|) \\
& <\frac{k_{4}^{*}[n t]}{n^{2 H}}\left(1+2 \omega^{2(H-1) / \alpha} \sum_{s=1}^{[n t]-1} s^{2(H-1)}\right) \\
& \sim \frac{2 k_{4}^{*} n t \omega^{2(H-1) / \alpha}}{n^{2 H}} \int_{0}^{n t} s^{2 H-2} \mathrm{~d} s \\
& =\frac{2 k_{4}^{*} t^{2 H} \omega^{2(H-1) / \alpha}}{2 H-1} \\
& <\infty
\end{aligned}
$$

for $k_{4}^{*}=\sum_{k=2}^{\infty}\left(C_{k}^{\iota}\right)^{2}<\infty$. Similarly, using (16), we have

$$
\mathrm{E}\left(e_{k}^{\iota}\left(V_{t}\right) e_{m}^{\iota}\left(V_{t+s}\right)\right) \geq \delta_{k}^{m} \rho_{1}^{2}(s)\left(\frac{1}{1+\iota}\right) \cdots\left(\frac{k-1}{k-1+\iota}\right),
$$

so, for $\left(C_{k}^{\iota *}\right)^{2}=\left(C_{k}^{\iota}\right)^{2}(1 /(1+\iota)) \cdots((k-1) /(k-1+\iota))$ and $k_{5}^{*}=\sum_{k=2}^{\infty}\left(C_{k}^{\iota *}\right)^{2}>0$, from (8),

$$
\begin{aligned}
\operatorname{var}\left(\frac{1}{n^{H}} \sum_{m=1}^{[n t]} \sum_{k=2}^{\infty} C_{k}^{\iota} e_{k}^{\iota}\left(V_{m}\right)\right) & \geq \frac{1}{n^{2 H}}\left(\sum_{k=2}^{\infty}\left(C_{k}^{\iota *}\right)^{2}\right) \sum_{m=1}^{[n t]} \sum_{s=1}^{[n t]} \rho_{1}^{2}(|m-s|) \\
& >\frac{k_{5}^{*}}{n^{2 H}}\left([n t]+2(1+\omega)^{2(H-1) / \alpha} \sum_{s=1}^{[n t]-1}([n t]-s) s^{2(H-1)}\right) \\
& \sim \frac{2 k_{5}^{*}(1+\omega)^{2(H-1) / \alpha}}{n^{2 H}} \int_{0}^{n t}(n t-s) s^{2 H-2} \mathrm{~d} s \\
& =\frac{2 k_{5}^{*} t^{2 H}(1+\omega)^{2(H-1) / \alpha}}{2 H(2 H-1)} \\
& >0,
\end{aligned}
$$

where we have used the fact that, for $s \geq 1, \alpha, \gamma, \omega>0$ and $\frac{1}{2}<H<1$,

$$
\left(1+\omega s^{\alpha}\right)^{\gamma(H-1) / \alpha}>\left(s^{\alpha}+\omega s^{\alpha}\right)^{\gamma(H-1) / \alpha}=(1+\omega)^{\gamma(H-1) / \alpha} s^{\gamma(H-1)},
$$

and Cauchy's integral test. Hence, in the case $\rho_{2}(s)=\rho_{1}^{2}(s), 0<\liminf _{n \rightarrow \infty} \operatorname{var}\left(D_{n t} / n^{H}\right) \leq$ $\lim \sup _{n \rightarrow \infty} \operatorname{var}\left(D_{n t} / n^{H}\right)<\infty$. 
Hence, we have proved Theorem 1 and further proved that, when $\rho_{2}(s)=\rho_{1}^{2}(s)$, so that Assumption 1 holds but $\sum_{s=1}^{\infty} \rho_{2}(s)=\infty, A_{n t} / n^{H}$ and $B_{n t} / n^{H}$ converge in mean square to 0 as $n \rightarrow \infty, C_{n t} / n^{H}$ converges weakly to the sum of [v] independent copies of the Rosenblatt process, and $D_{n t} / n^{H}$ has, for each fixed $t$, variance bounded away from 0 and $\infty$.

\section{Discussion}

We have constructed a long-range dependence (LRD) and asymptotically self-similar activity time process $\left\{T_{t}\right\}$ which has inverse gamma distributed increments with noninteger distributional parameters. This process naturally extends that considered in Heyde and Leonenko (2005) to the context of a subordinator model for financial assets with marginal $t$-distribution, which was restricted to integer parameter values, and, thus, supports the use of the $t$-model for actual financial data.

We also note that there are other methods of constructing activity time processes with LRD and an asymptotically self-similar limit. For example, Leonenko et al. (2012) constructed gamma and inverse Gaussian processes, based on a superposition of Ornstein-Uhlenbecktype processes, which converge to Brownian motion (in the case of a finite superposition) or fractional Brownian motion (in the case of an infinite superposition). Finlay and Seneta (2007) also reviewed a number of different approaches to constructing activity time processes.

\section{Acknowledgement}

The authors are grateful to N. N. Leonenko for helpful discussions regarding the $D_{n t}$ process.

\section{References}

Finlay, R. and Seneta, E. (2006). Stationary-increment Student and variance-gamma processes. J. Appl. Prob. 43, 441-453. (Correction: 43 (2006), 1207.)

Finlay, R. And SEneta, E. (2007). A gamma activity time process with noninteger parameter and self-similar limit. J. Appl. Prob. 44, 950-959.

Finlay, R., Fung, T. and Seneta, E. (2011). Autocorrelation functions. Internat. Statist. Rev. 79, $255-271$.

Gneiting, T. (2000). Power-law correlations, related models for long-range dependence and their simulation. J. Appl. Prob. 37, 1104-1109.

Griffiths, R. C. (1969). The canonical correlation coefficients of bivariate gamma distributions. Ann. Math. Statist. 40, 1401-1408.

Heyde, C. C. And Leonenko, N. N. (2005). Student processes. Adv. Appl. Prob. 37, 342-365.

LEONEnKo, N. (1999). Limit Theorems for Random Fields with Singular Spectrum. Kluwer, Dordrecht.

Leonenko, N. N., Petherick, S. And Sikorskit, A. (2012). Fractal activity time models for risky asset with dependence and generalized hyperbolic distributions. Stoch. Anal. Appl. 30, 476-492.

TAQQU, M. S. (1975). Weak convergence to fractional Brownian motion and to the Rosenblatt process. Z. Wahrscheinlichkeitsth. 31, 287-302. 